\title{
Modeling Outflows from Young Stars
}

\author{
Hsien Shang \\ Institute of Astronomy and Astrophysics, Academia Sinica, Taiwan
}

\begin{abstract}
We have constructed the foundations to a series of diagnostics methods to probe the jet phenomena in young stars as observed at various optical forbidden lines and radio wavelengths. We calculate and model in a self-consistent manner the physical and radiative processes, which arise within an inner disk-wind driven magnetocentrifugally from the circumstellar accretion disk of a sun-like star. Comparing with real data taken at high angular resolution, our approach will provide the basis of systematic diagnostics for jets and their related young stellar objects, to attest the emission mechanisms of such phenomena. Such approach can help bring first-principle theoretical predictions to confront actual multi-wavelength observations, and will bridge the link between complex numerical simulations and observational data. Analysis methods discussed here are immediately applicable to new high-resolution data obtained with HST, Adaptic Optics, and radio interferometry.
\end{abstract}

\section{Introduction}

Perhaps the most important and yet most debated question for YSO jets both theoretically and observationally is what makes the jets as we see them. This question remains, and becomes even more pressing after significant advances made in all aspects over the past decade. HST and Adaptic Optics have revealed images and kinematical information to a great detail: jets can be seen at optical wavelengths down to a few $\mathrm{AU}$ from the sources, and knots can be resolved into bow shocks or internal working surfaces. On the theoretical fronts, models based on first-principles of physics have reached consensus that the likely driving mechanism is magnetocentrifugal winds from the surroundings of the central stars driven by a combination of magnetic field and rotation. Numerical simulations have reached the level that the majority of dynamical effects: launching, acceleration, and propagation have been individually investigated. Regardless of the approaches, all lead to the same ultimate question. What is the emission mechanism in jets that make them as they have been observed?

If a unified picture can emerge, how do jets appear out of the winds driven magnetocentrifugally at more than $30^{\circ}$ from the axes? A very noticeable feature of the MHD processes that could collimate the winds on the large scale toward the axes of rotation is the density collimation. This density collimation arises because the wrapped-up magnetic field lines of the winds produce density profiles stratified cylindrically with horizontal distances from the rotation axes. How the density structures of MHD winds can play the trick optically through photons 
is the major challenge facing any theoretical MHD wind model whose mattercarrying streamlines are slowly collimated over the scale that jets have been observed. Is it the density collimation rather than the streamline collimation that makes the jets jet-like? How does such "optical illusion" get to illude human eyes into believing the underlying driving mechanisms? In this proceedings, we review the modeling and interpretation for the emission mechanisms of YSO jets based on the $\mathrm{X}$-wind model.

\section{Basic Picture of The $\mathrm{X}$-winds}

The $\mathrm{x}$-wind model is rooted in an evolutionary picture of how a new born star interacts with its surroundings during the important phases of accretion. A new born star can develop its magnetosphere as soon as it becomes sufficiently convective through the dynamo action. The magnetic fields thus generated can interact with the accretion disk where the matter comes in to feed the star. The disk is truncated by the magnetosphere and pushed away from the star, and the magnetic fields being pressed against the disk are pinched at the inner edge of the disk at the "x-point". When the field is pinched strongly enough to truncate the disk, simultaneous outflow and inflow occur along the pinched field lines from the x-point to form an "X-wind" and a "funnel flow" that carries matter to accrete onto the star. The structure of such a "fan" of magnetic fields around the x-point is shown in Fig. 9 of Shu et al (1994). In steady state, fields having foot points on both the star and the disk will synchronize the rotation of the star and the inner edge of the disk $\left(R_{x}\right)$.

On the large scale, the hoop stresses associated with the magnetic fields slowly re-direct the matter flowing along the poloidal magnetic field ("streamlines") into the spin axis of the system over a long length scale by magnetic collimation. The streamlines remain very divergent, and only approach vertical at infinity. The wrapped-up field lines, on the other hand, make matter density stratified cylindrically with horizontal distances $(\varpi)$ from the rotation axes. Because $\mathrm{x}$-wind is driven from a very small region near the $\mathrm{x}$-point and completely expands to fill the space, the effect of wrapped fields and the density collimation works the strongest. The strong cylindrically stratified density profiles can be seen very clearly from a few AU from the star, and most prominent when viewing at hundreds and thousands of AU away from the source. This is the density collimation that is at work, in addition to streamline collimation in the MHD wind models. Figure 1 of Shang et al (1998) demonstrated such density contours on top of the background still very divergent streamlines. This forms the basis of "optical illusion" when the optical emission lines are properly excited (Shang et al. 1998, hereafter SSG), as first proposed by Shu et al (1995).

Near the base of the wind and only few stellar radii from the star, the magnetic field varies from a closed geometry to opened field at the boundary between the $\mathrm{x}$-wind and the deadzone, and in-between the funnel flows where the field mediates between the closed dipole (multipoles) and the inner edge of the disk. These kinks have the Y-shape like the helmet streamers found on the solar magnetic loops. These magnetic loops and helmet streamers are the sites for x-ray generation, soft and hard, in quiescence and in flares. When enhanced magnetic activities associated with wrapped up fields and reconnection take 
place, occurring on short time scales, strong x-ray flares arise and the events have been seen from a few protostars. UV photons, on the other hand, are generated from the hot spots where the funnel flows strike the surface of the young stars. Both radiation mechanisms are capable of ionizing and heating the wind flow with favorable penetration near the base of the wind from a slightly elevated angle above the disk.

\section{The SGSL Approach of Heating and Ionization of X-winds}

Shang et al (2002, hereafter SGSL) developed an integrated approach that put together all major physical processes that occur in the neighborhood of the star, the disk, and the $\mathrm{x}$-wind. The SGSL treatment included both internal mechanisms in a wind launched by MHD mechanisms via a star-disk interaction, and external disturbances that could go on top of the background flow. Table 1 in SGSL summarizes the processes that have been built into the approach based on predominately $\mathrm{H}$ atoms: ionizations due to the $\mathrm{x}$-rays, Balmer continuum, $\mathrm{H}^{-}$ detachment, and by collisions; and heating due to X-rays, ambipolar diffusion, Balmer photoionization of $\mathrm{H}, \mathrm{H}^{+}+\mathrm{H}^{-}$neutralization, $\mathrm{H}^{-}$photodetachment, and the mechanical contribution from the environment. The ionization is primarily offset by radiative recombination, and the adiabatic expansion cools very efficiently with the rest: $\mathrm{H}^{-}$radiative attachment, recombination of $\mathrm{H}^{+}$, Ly $\alpha$, collisional ionization, and line cooling from the heavy elements.

To mimic the effects of time-variabilities often seen with jets and knots, we adopt here the phenomenological expression for the volumetric rate of mechanical heating (cf. eq [5-2] of SGSL):

$$
\Gamma_{\text {mech }}=\alpha \rho \frac{v^{3}}{s} .
$$

where $\rho$ and $v$ are the local gas density and flow velocity in an inertial frame at rest with respect to the central star, and $s$ is the distance the fluid element has travelled along a streamline in the flow. The coefficient $\alpha \geq 0$ phenomenologically characterizes the magnitude of disturbances, possibly magnetic in origins. A choice of $\alpha \ll 1$ indicates that only a small fraction of the mechanical energy contained in the shock waves and turbulent cascades is dissipated into heat when integrated over the flow volumes of interest at the characteristic distances $s$. Small values of $\alpha$ are required both for self-consistency of a cold flow, and in order to have a regime of weak disturbances on top of a background flow whose properties have been preserved. In the situations where strong disturbances occur, $\alpha$ could and should be very localized in nature, depending on the local dissipation of shocks and turbulence in the neighboring fluid cells. As seen in most jet images, the jets are knotty by appearance, and multi-epoch observations also reveal time-variabilities over the period of various observations. All these possible sources of variabilities and disturbances on the flow add up to an averaged $\alpha$, for modelling purposes. For example, $\alpha \approx 0.002$ would suggest a velocity variation less than $5 \%$. Given a typical jet velocity of $300 \mathrm{~km} / \mathrm{s}$, this would imply a weak shock of $15 \mathrm{~km} / \mathrm{s}$ decaying over the jet length. With all the ingredients combined, the thermal profiles thus produced are self-consistent with 
the underlying dynamical properties, stellar parameters, chemistry, heating and ionizing sources in the framework of star-disk interacting systems.

\section{Observational Diagnostics in Optical Forbidden Lines}

The studies based on SGSL approach suggest that the X-rays and UV photons derived from the accretion processes in young stars could contribute to most of the ionization down deep in the acceleration zone, and that X-rays and recombination on the larger scale, could determine the residual level of ionization in wind and the jet proper over several thousand AU of scale. The level of a few percent and the pattern of reaching a plateau then decaying along the length of jets by recombination is consistent with the trend usually inferred from groundbased and space observations (e.g. Bacciott \& Eislöffel 1999, Bacciotti et al 2000). Figures 1, 2, and 3, in SGSL, illustrate such profiles of ionization on the bottom panels. The top panels in these figures show temperature behaviors for $\alpha=0,0.001$, and 0.002 , respectively. The choice of $\alpha \approx 0.002$ for a slightly revealed fiducial YSO source is the minimum to maintain a temperature high enough in wide enough emitting areas to excite the red forbidden lines. With a temperature around $8,000-10,000 \mathrm{~K}$, the ionization fraction $x_{e}$ holds up very well on the level of few percent on the largest scale. Closer in, $x_{e}$ could rise up to a few tens of percent. Observationally, the highest jet ionization fraction have also been inferred to be from close to the sources (e.g. Lavalley-Fouquet et al 2000 , Bacciotti et al 2002). Again, qualitatively, the range obtained from model calculations are within the observational range of 0.02-0.5 (Ray \& Bacciotti 20003).

Synthetic images of fiducial sources can be made by calculating the emission profile with the excitation condition expressed by $x_{e}$ and $T$ (e.g. Fig. 3 of SGSL), and with the cylindrically stratified density structures calculated for with the specific stellar parameters (see Fig. 1 of SSG). The overall brightness in the synthetic image with a temperature profile $T \approx 8000 \mathrm{~K}$ or higher in large enough emitting areas, indeed can be established by a small parameter $\alpha$. Looking at the brightness profile, the projection of a cone-shaped opening near the base actually has appeared, indicating a possible jet opening angle could exist in the optical image out of a wind whose streamlines are launched into all solid angles. The self-consistent excitation profile has changed substantially from the pure column density effect illustrated in Fig. 2 of SSG and suggests that excitation plays crucial roles in how the structure of jets could be revealed. Higher along the length of a jet, the density stratification gradually dominates, and the jetlike appearance stands. This suggests that the excitation conditions reached in jets and winds can completely make over how jets are to be seen optically, and that the effects of heating mainly contribute to change of excitation on top of background flow that could be closely modelled by steady-state values. The optical jets could indeed be a manifestation of "optical illusion" by the interplay of excitation and density collimation.

The line ratio plots are the best diagnostic tools to ascertain whether such approach could effectively and accurately capture the excitation and physical conditions embedded in jets from optical forbidden line. In Fig. 5 of SGSLwhere the line ratios of S[II]6716/S[II] 6731 and S[II] $6731 / O[I] 6300$ are plotted 
against each other. The former is an indicator of the electron density, whereas the latter is an indicator of both the electron density $\left(n_{e}\right)$ and temperature. A cross-correlation plot of these line ratios will uniquely determine the excitation condition by the location on the plot. The small blue dots plotted are each theoretical data point for each "pixel" for the images of S[II] and O[I] jets in Fig. 4 of SGSL. The red stars are data compiled by Raga et al (1996) from ground based observations prior to 1995 . Up to the date the plot was made, the pink circles were points for DG Tau with Adaptic Optics (data taken from Fig. 3 of Lavalley-Fouquet, Cabrit, \& Dougados 2002). One can also compare with the AO data for RW Aur (Dougados et al 2002), and the points would fall very well within the coverage. One very noticeable feature of this plot is that the theoretical data dots pretty much encompass all of the observational data points to date. With only few points, whose sources are known to be strong shock excited objects (in knots or bow shocks), lying outside of the loci traced by the shape of potential curves, the physical and excitation condition reached in $\mathrm{x}$-wind jets for a slightly revealed source indeed captures the majority and average conditions inferred from optical jets.

What does such comparison of theoretical and observational data suggest for YSO jets? The treatment of weak shocks works quite well on top of a steadystate background flow. This also suggests that in fact the radiation from jets are mostly excited by weak shocks on average. A single $\mathrm{x}$-wind jet modelled for the slightly revealed YSOs could generate the range of physical and excitation conditions inferred from $\left(n_{e}, x_{e}, T\right)$ of the most studied $\mathrm{HH}$ objects. This also implies that one perhaps can not attempt to label the jets with one single set of numbers $\left(n_{e}, x_{e}, T\right)$ because the system is quite complex. It would require fits from multiple shock curves of various pre-shock densities, velocity variations, and strengths of magnetic fields (e.g. Fig 4. in Dougados et al. 2002). The theoretical models can give a more complete view, and help probe the varieties of conditions.

\section{Observational Diagnostics in Radio Continuum}

Radio jets are elongated, jet-like structures seen at subarcsecond scale. They usually have good alignment with large-scale outflows, usually identifiable with the youngest deeply embedded stellar objects, and have partial association with optical jets or Herbig-Haro objects. In a few YSO sources, the optical jets trace material at scales of several hundred AU and larger, but near the base, small radio jets from ionized material are present very close to the (projected) bases of the optical jets. The typical fluxes from radio jets are weak, around 0.2-2 $\mathrm{mJy}$, and usually are characterized by a small spectral index, $S_{\nu} \propto \nu^{i}$, where $i \geq-0.1$. A complete list of radio jets from both low-mass and high-mass sources, and their respective properties $\left(S_{\nu}, i\right.$, etc) can be found Table 2 in Anglada (1996).

We are interested in studying low-mass young stellar sources where radio jets have been observed. One of these sources is the archetype L1551-IRS5 found in the Taurus molecular cloud at a distance of $140 \mathrm{pc}$. This source is one of the most studied outflow sources. It has a massive molecular outflow (e.g. Snell, Loren \& Plambeck 1980) driven by an atomic stellar wind (e.g. Giovanardi et al. 2000). L1551-IRS 5 has a bolometric luminosity of $33 L_{\odot}$ and was identified 
as a Class I source with a total wind mass loss rate of $\sim 2 \times 10^{-6} M_{\odot} y r^{-1}$, obtained from the HI observations (Giovanardi et al 2000). Recently, Rodríguez et al. (2003) used VLA and Pie Town antenna observations with an angular resolution of 0.1 (14 AU), and found radio jets from the now-identified binary (e.g. Rodríguez et al 1998) at the origin of the larger scale $(\sim 1 ")$ jets observed in both optical and NIR wavelengths (Fridlund \& Liseau 1998; Itoh et al. 2000). The level of radio emission from both jets is $\sim 4 \mathrm{mJy}$, and half of which comes from two compact jet cores.

The SGSL excitation profiles of temperature and ionization, and the electron density distribution for $\dot{M}_{w}=1 \times 10^{-6} M_{\odot} \mathrm{yr}^{-1}, \alpha=0.01$ have resemblance to the profiles shown in Fig. 3 of SGSL. At scales relevant to radio jets, the ionization fraction is on the level of few percent, and this is by a factor of a few reduced from the fiducial case in SGSL at $\dot{M}_{w}=3 \times 10^{-8} M_{\odot} / \mathrm{yr}$. The temperature on the axis near the center is around $10,000 \mathrm{~K}$, as has been inferred for most radio jets. Within $20 \mathrm{AU}$ from the z-axis, electron densities as high as $10^{4}-10^{6} \mathrm{~cm}^{-3}$ can be clearly identified on the scale of $100 \mathrm{AU}$. Very close in ( $\leqslant 10 \mathrm{AU}$ ), the electron densities could raise even higher to $10^{7}-10^{8} \mathrm{~cm}^{-3}$. As the resolution improves, the higher the electron densities can get closer to the source.

An intensity map at $3.6 \mathrm{~cm}$ based on the above mentioned profile is shown in Figure 1a. The contour levels are plotted at powers of 10. The elongated contours show clearly the collimation due to cylindrically stratified electron density profiles, and can be traced down to 10 AU level. This theoretical intensity map has a lot of fine structure that is yet to be detected by the observation beyond the current 0 ". 1 limit. For radio emissions from the jet to be detected by an instrument like VLA at its highest sensitivity and angular resolution, enough total $n_{e}$ and higher mass loss rates with the combinations of parameters are required to produce the right order of magnitudes in total emitted fluxes. The case shown has a total flux from half of jet lobe of $1.14 \mathrm{mJy}$.

A relation of $S_{\nu}-\nu$ (as in Fig. 1b) best illustrates the behavior of total flux with radio frequencies and the spectral index $i$ at various mass loss rates. For mass loss rates lower than $3 \times 10^{-7} M_{\odot} \mathrm{yr}^{-1}, S_{\nu} \propto \nu^{-0.1}$, indicating pretty much transparent emission. The spectra turn over as the mass loss rates progress up to around $\dot{M}_{w} \approx 3 \times 10^{-7} M_{\odot} \mathrm{yr}^{-1}$, at which the indices change from approximately 0.04 , to -0.1 , at around $8.3 \mathrm{GHz}$, suggesting at this mass loss rate emission from optically thick region starts to appear. For mass loss rates higher than the "cross-over" mass rate $\dot{M}_{w}$, the index is approximately $0.3-$ which is very close to what has been inferred for L1551.

The extension of the SGSL approach to radio frequencies adds another dimension to the diagnostic tools built upon the SGSL approach and the x-wind model. The radio jets have the following unique advantages. Radio free-free emission at centimeter wavelength is free of dust extinction effects, and no obstruction from disks that the optical and infrared observations and diagnostics usually suffer from. The radio jets have strong connections with deeply embedded protostars and optical jets (if any) from those sources are not easily seen. These make the radio free-free emission a powerful diagnostic tool to probe deeper toward exciting sources. 

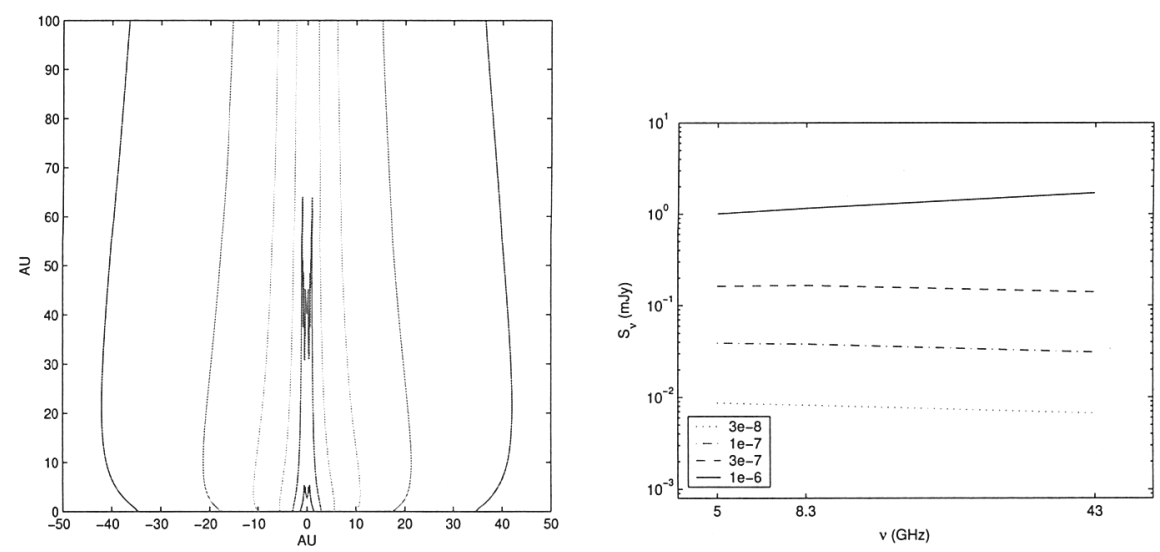

Figure 1. a. Intensity map made for $\dot{M}_{w}=1 \times 10^{-6} M_{\odot} / \mathrm{yr}$, and $\alpha=0.01$. The contours are plotted at powers of 10 , at scales relevant to radio jets. This figure shows more detail than current resolution limit allows one to see. b. A plot of the relationship $S_{\nu}-\nu$ for the four different mass loss rates: $3 \times 10^{-8}, 1 \times 10^{-7}, 3 \times 10^{-7}$, and $1 \times 10^{-6}$ $M_{\odot} / \mathrm{yr}$.

\section{Summary and Outlook}

What have we learned with modeling and diagnostic tools based on the $\mathrm{x}$-wind model? First and foremost, $\mathrm{x}$-wind jets with proper heating and ionization can provide an explanation for the general properties observed to date from radio free-free emissions from the youngest low-mass stars to optical forbidden lines from T-Tauri stars. The extension of SGSL approach to protostars strongly suggests a connection between winds and jets from CTTS to outflows from protostars. First-principle theoretical approach such as SGSL can help provide the basis of systematic diagnostics, and probe the emission mechanisms. When going to high-angular resolution, theoretical modeling and predictions can serve as motivations leading to smaller and smaller scales.

Then we ask the question, what can we do for studying jets at high angular resolution? We have already had adaptic optics, and the space telescope. We have radio interferometry already working and more on the way, and optical interferometry in planning. With all these telescopes pushing into higher and higher angular resolution, we will need multiwavelength measurement, and longer and longer integration time.

\section{References}

Angalad, G. 1996, in Radio Emission from the Stars and the Sun, eds. A. R. Taylor \& J. M. Paredes, (PASP: San Francisco), 3

Bacciotti, F. \& Eislöffel, J. 1999, A\&A, 342, 717 
Bacciotti, F.; Mundt, R.; Ray, T. P.; Eislffel, J.; Solf, J.; Camezind, M. 2000, ApJ, 537: L49-L52

Bacciotti, F.; Ray, T. P.; Mundt, R.; Eislöffel, J.; Solf, J. 2002, ApJ, 576: 222

Dougados, C.; Cabrit, S.; and Lavalley-Fouquet, C. 2002, RevMexAA (Serie de Conferencias), 13, 43

Dougados, C.; Cabrit, S.; Lavalley, C.; Mnard, F. 2000, A \& A, 357: L61-L64

Fridlund, C. V. M. and Liseau, R. 1998, ApJ, 499, L75

Giovanardi, C., Rodríguez, L. F., Lizano, S., \& Cantó, J. 2000, ApJ, 538, 728

Itoh, Y., et al. 2000, PASJ, 52, 81

Lavalley-Fouquet, C., Cabrit, S., Dougados, C. 2000, ApJ, 356, L14

Raga, A., Bhm, K.H., Canto, J., 1996, RevMexAA 32, 161

Ray, T., \& Bacciotti, F. 2003, RevMexAA (Serie de Conferencias), 15, 106

Rodriguez, L. F., et al. 1998, Nature, 395, 355

Rodriguez, L. F., Porras, A., Claussen, M., Curiel, S., Wilner, D., and Ho, P. 2003, ApJL, 586, L137

Ruden, S. P., Glassgold, A. E., Shu, F. H. 1990, ApJ, 361: 546-569

Shang, H., Glassgold, A. E., Shu, F. H., and Lizano, S. 2002, ApJ, 564: 853-876 (SGSL)

Shang, H., Lizano, S., Glassgold, A., and Shu, F. H. 2003, ApJ, to be submitted

Shang, H., Shu, F. H., and Glassgold, A. E. 1998, ApJ, 493: L91-L94 (SSG)

Shu, F., Allen, A.; Shang, H.; Ostriker, E.; Li, Z.-Y. 1999, in The Origin of Stars and Planetary Systems. Edited by Charles J. Lada and Nikolaos D. Kylafis. Kluwer Academic Publishers, 193

Shu, F., Najita, J., Ostriker, E., and Shang, H. 1995, ApJL, 455, 155

Shu, F., Najita, J., Ostriker, E., Wilkin, F., Ruden, S., Lizano, S. 1994, ApJ, 429: 781-796

Woitas, J.; Ray, T. P.; Bacciotti, F.; Davis, C. J.; Eislffel, J. 2002, ApJ, 580, 336-342 